\title{
The Impact of Anxiety on Chronic Obstructive Pulmonary Disease
}

\author{
Hoda Mojazi Amiri, Khalid Monzer, Kenneth Nugent ${ }^{*}$ \\ Department of Internal Medicine, Texas Tech University Health Sciences Center, Lubbock, USA \\ Email: "kenneth.nugent@ttuhsc.edu
}

Received July $9^{\text {th }}, 2012$; revised August $10^{\text {th }}, 2012$; accepted September $4^{\text {th }}, 2012$

\begin{abstract}
Patients with COPD have chronic respiratory symptoms and significant physical limitations secondary to abnormal pulmonary function. These patients often have significant comorbidity, including psychiatric disorders such as anxiety and/or depression. The frequency of anxiety and generalized anxiety disorder (GAD) in patients with COPD significantly exceeds the frequency in the general population. GAD reduces the quality of life in these patients and potentially contributes to acute flares in their chronic lung disease. Most neurobiological models for anxiety involve the limbic system and amygdala. Stimulation of these areas results in fear and anxiety. The sense of dyspnea (an unpleasant sensory perception of difficult breathing) also stimulates the limbic system, including the amygdala. Consequently, episodes of dyspnea could contribute to the development of anxiety in these patients through kindling phenomena. This could evolve to the point that the patient has chronic anxiety which is maintained by the ongoing sense of dyspnea secondary to chronic lung disease. Pulmonary rehabilitation improves the overall quality of life in these patients and reduces respiratory symptoms, including dyspnea. Patients who do not respond to pulmonary rehabilitation or who have more severe GAD may need chronic medications, such as selective serotonin re-uptake inhibitors. These patients will also benefit from psychological and psychiatric evaluation and care.
\end{abstract}

Keywords: Generalized Anxiety Disorder; COPD; Acute Flare; Treatment

\section{Introduction}

Patients with chronic obstructive pulmonary disease (COPD) have a chronic irreversible lung disease characterized by airflow limitation on spirometric testing. The pathological changes typically include both chronic bronchitis and emphysema. These patients have chronic respiratory symptoms, reduced quality of life, physical limitations, disability, increased medical costs, frequent hospitalizations, and premature death. This disease occurs worldwide and usually occurs in chronic cigarette smokers. Patients frequently present after the age of 50 and are at risk for other chronic comorbidity, including cardiac disease, hypertension, diabetes, and malignancy. Patient management should focus on smoking cessation, improvement in lung function when possible, limiting the deterioration in lung function, preventing acute exacerbations, and managing comorbidity. These patients frequently have anxiety and/or depression, and some clinical literature suggests that anxiety increases the frequency and/or severity of exacerbations. This review will focus on the association between anxiety and COPD.

\section{Diagnostic Criteria for Anxiety}

The diagnosis of anxiety in patients with COPD requires a careful clinical assessment. Physicians can use checklists, such as the Beck Anxiety Inventory (BAI), The Taylor Manifest Anxiety Scale, or the Zung Self Rating Anxiety Scale, or structured clinical interviews. The Beck Anxiety Inventory has 21

${ }^{*}$ Corresponding author. categories which are rated 0 to 3 , with 3 indicating that the symptom "bothered the patient a lot" (Beck \& Steer, 1996). The scores are summed and the patient is placed into categories of low, mild, moderate, or severe anxiety. This scale is frequently used in clinical and population studies. Structured clinical interviews make use of the Diagnostics and Statistics Manual for Mental Disorders (DSM-IV) (American Psychiatric Association, 2000). The diagnosis of a General Anxiety Disorder (GAD) based on the DSM-IV has strict diagnostic criteria, including excessive anxiety and/or worry for at least six months, difficulty in controlling the worry, and at least three of six listed symptoms. This anxiety cannot be explained by another Axis 1 psychiatric disorder, by substance abuse, or by a general medical condition, and it must cause clinically significant distress or impairment in social, occupational, or other areas of functioning. The use of structured interviews typically requires training in either psychology or psychiatry, and these are not used by most physicians in their routine practice. The BAI includes somatic symptoms, such as dizziness, palpitations, shortness of breath, and indigestion; the DSM-IV diagnostic criteria include symptoms, such as restlessness, fatigue, difficulty concentrating, irritability, muscle tension, and sleep disturbance. These somatic complaints and psychological symptoms may occur in patients with COPD as a consequence of their underlying chronic lung disease and/or treatment and might not necessarily represent a separate diagnosis, such as GAD. Consequently, deciding whether a patient with COPD has psychological distress as a consequence of underlying respiratory symptoms and impairment or has a separate independent disorder is difficult and warrants consideration when reviewing studies on the 
prevalence of anxiety in COPD. In addition, this distinction has implications for both treatment and outcome.

\section{Anxiety Prevalence in COPD}

Several studies have reported a high prevalence of anxiety in patients with COPD, but the rates vary widely in these studies (Di Marco et al., 2006; Eiser et al., 1997; Kunik et al., 2005). The prevalence of moderate to severe anxiety ranges from $2 \%$ 96\% (Hynninen et al., 2005; Hill et al., 2008; Brenes, 2003). This variation may reflect differences in methodologies and criteria used to diagnose anxiety, such as self-report vs structured clinical interviews. The lowest prevalence $(2 \%)$ was reported by Light et al. who used State Trait Anxiety Inventory and considered scores two standard deviations above the mean as positive (Light et al., 1985). The highest prevalence (96\%) was reported by Borak et al who used self-reports based on the Taylor Manifest Anxiety Scale in COPD patients with chronic respiratory failure (CRF) (Borak et al., 1991). Kunik et al. reported the largest study on the prevalence of anxiety disorder in patients with COPD (Kunik et al., 2005). These authors first used the Primary Care Evaluation of Mental Disorders (PRIME-MD) screening questions to screen 1334 patients with a chronic breathing problem for anxiety and/or depression. Nine-hundred and ninety-five (75\%) patients tested positive for anxiety. The authors then used DSM-IV based structured interviews to confirm the presence of anxiety or depression in patients with COPD who tested positive on PRIME-MD. Two hundred and four patients underwent DSM-IV interviews, and $103(50.5 \%)$ had GAD.

Recent review articles have reported the prevalence of GAD is $10 \%-15.8 \%$ in COPD patients compared to $3.6 \%-5.1 \%$ in the general public (Brenes, 2003). The prevalence of panic disorder is $8 \%-67 \%$ in patients with COPD (Hynninen et al. 2006). The 12 month and lifetime prevalence of panic disorder in the general public in the United States is $2.7 \%$ and $4.7 \%$, respectively (Kessler et al., 2005). These studies confirm that anxiety disorders are more common in COPD patients than the general population. In addition, the reverse situation is also true. Zandbergen reported that the lifetime prevalence of respiratory disorders in patients with panic disorder is $47 \%$, which is significantly higher than in patients with obsessive-compulsive disorders $(13 \%)$ or eating disorders (13\%) (Zandbergen et al., 1991). These studies suggest that COPD and anxiety have an unusual linkage, that COPD contributes to the pathogenesis of $\mathrm{GAD}$, or that the symptom profile in COPD leads to misdiagnosis of GAD.

Ferguson and coworkers addressed this issue in a study which involved 202 military veterans with COPD (Ferguson et al., 2006). These veterans completed BAI, BDI, and MiniMental state examinations. They then underwent structured interviews using DMS- IV criteria to determine whether or not they had anxiety or depression. Eighty-six percent of the participants exceeded the cut-off score for depression based on the BDI, and $77 \%$ exceeded the cut-off score for anxiety based on the BAI. Based on structured clinical interviews, 30.2\% met diagnostic criteria for depressive disorder, and $31.7 \%$ met criteria for an anxiety disorder. Confirmatory factor analysis suggested that a two factor model which included depression and anxiety best fit the data. Therefore, these authors concluded that somatic symptoms in patients with COPD were good indicators of depression and/or anxiety. However, they did not exclude the possibility that these diagnoses represented a mood disorder associated with a general medical condition. Making this distinction remains quite difficult even in a study like this and probably requires evaluations by both a psychiatrist and a pulmonary physician to determine whether or not the mood disorder symptoms represent an independent medical disorder. However, the main conclusion from this study is that mood disorder symptoms are quite common in these patients and potentially have important effects on disease course and quality of life.

\section{Association with Acute Flares}

Most patients with COPD have episodic increases in their symptoms which are referred to as acute exacerbations of COPD. These patients typically have increased cough, increased sputum production, and increased shortness of breath. They often require changes in their management, hospitalizetion, and occasionally mechanical ventilation for respiratory failure. Viral infections, bacterial infections, environmental pollution, and other acute medical disorders, usually cause acute flares in COPD. A few studies have examined the effect of psychiatric disorders on the risk of COPD exacerbation, and these are reviewed in this section.

In a meta-analysis of nine prospective studies, Laurin et al. reported patients with anxiety had a higher risk for out of hospital treated COPD exacerbations (RR: 1.23; 95\%CI: 1.03 1.47) (Laurin et al., 2012). However, anxiety had no effect on risk for in-patient treated COPD exacerbations (RR: 1.05 95\%CI: $0.92-1.19$ ). They suggested that fear and increased symptom awareness in patients with anxiety caused "early interventions" in these patients and prevented exacerbation progression which would require in-patient treatment. $\mathrm{Xu}$ et al. measured depression and anxiety with the Hospital Anxiety and Depression Scale (HADS) at baseline in 491 patients with stable COPD (Xu et al., 2008). These patients were then followed for 12 months to determine the frequency of COPD exacerbation and hospitalizations. Patients with probable anxiety (HADS anxiety score $>11$ ) were more likely to have a longer duration of event-based ( $\geq 1$ symptom worsening plus $>1$ change in regular medications) exacerbations than patients without anxiety (HADS score $<7$ ). Also, patients with anxiety were more likely to have symptom-based (worsening of $>1$ key symptom) exacerbations than those without anxiety, but the number of hospitalizations was not significantly different between the two groups. Conversely, Yohannes et al. reported that anxiety score is independently associated with frequency of hospitalization in a group of elderly patients with COPD (Yohannes, Baldwin, \& Connolly, 2000). Variation in study methods, including the definition of COPD exacerbation, criteria used to diagnose anxiety, and different patient populations, likely influenced the results in these studies.

Laurin suggested that the relationship between anxiety and COPD exacerbation was explained by activation of hypothalamic-pituitary-adrenal pathway axis and increased systemic inflammatory response, by low self-confidence which in turn causes worse coping and self-care behavior, and by fear and worry which could increase the awareness of symptoms and symptom reporting (Laurin et al., 2012). In summary, anxiety appears to increase the risk of acute exacerbations in patients with COPD. Early diagnosis and treatment of anxiety in patients with COPD could reduce somatic symptoms and possibly decrease the frequency of exacerbations. 


\section{COPD and Anxiety-Neuroendocrine and Drug Effects}

Patients with GAD have greater sensitivity to bodily sensations than control patients, and this contributes to their exaggerated responses to stress. Patients with COPD and hypoxemia have marked peripheral sympathetic activation, and this activity decreases after the correction of the hypoxemia with supplemental oxygen (Andreas et al., 2005; Wennlund \& Carlstrom, 2000; Heindl et al., 2001). These patients also have impaired autonomic heart rate control, probably explained by cardiac sympathetic nerve activation, and an activated rennin-angiotensin system. During acute flares they have increased levels of norepinephrine. In summary, patients with COPD have chronic sympathetic activation with increased responses during acute changes in disease status. This stress causes somatic symptoms, contributes to cardiovascular morbidity, and would increase symptoms in patients with comorbid anxiety.

Patients with COPD usually take bronchodilators and antiinflammatory drugs. Beta agonist bronchodilators are used in patients with COPD for both acute symptom relief and chronic symptom management. These drugs frequently cause tachycardia, palpitations, nervousness, tremor, and dizziness (Salpeter, Ormiston, \& Salpeter, 2004). Theophylline (a weak bronchodilator) has a similar side effect profile and can cause tremor, irritability, restlessness, tremor, and insomnia. Systemic corticosteroids are used during acute flares for anti-inflammatory effects and cause insomnia, nervousness, and mood swings. Therefore, these drugs have frequent side effects which mimic the somatic symptoms associated with GAD and could increase the severity of non-respirtory symptoms in these patients. Consequently, patients with COPD and GAD need careful medication reviews to determine whether or not some somatic symptoms reflect potentially avoidable drug side effects. Both sympathetic stress associated with acute flares and drug side effects could contribute to the development of anxiety through kindling which is discussed below in the pathogenesis section.

\section{Pathogenesis of Anxiety}

Patients with chronic anxiety have exaggerated responses to life stresses. This likely reflects abnormal function in regions of the brain associated with the detection and response to external dangers and involves both biological and social-environmental factors (Jetty, Charney, \& Goddard, 2001). These regions either respond to stress with increased stimulation of the limbic system (i.e., have a low activation threshold), or have an excessive response to potential danger, or both. Eventually this arousal state in GAD becomes chronic and is either autonomous or sensitive to very mild stresses. These responses involve the limbic system in the central nervous system, and this includes both cortical structures (the hippocampus, the insular cortex, the orbital frontal cortex, and other regions) and subcortical structures (the hypothalamus, the amygdala, and other nuclei). The limbic system is involved in both fear and anxiety responses and in emotional memory. There are several neurobiological models which potentially explain anxiety and generate clinical hypotheses about its pathogenesis. The fear circuitry model is centered in the amygdala (Jetty, Charney, \& Goddard, 2001). Animals, including humans, need to be able to sense danger; sensory inputs into the limbic system activate this system when danger is present. This results in the behavioral changes and physiologic changes associated with fear. These physiologic responses largely involve the autonomic nervous system and the endocrine system. The amygdala has an important role in this system since it coordinates behavioral, autonomic, and endocrine responses to environmental stimuli, especially those that have emotional content, through connections to the hypothalamus, brainstem, and cerebellum. Lesions in amygdala reduce responses to stress; stimulation of the amygdala produces behavioral arousal and can produce rage reactions. The fear circuitry model explains some of the symptoms associated with the generalized anxiety disorder, including hyperarousal, increased motor tension, stress sensitivity, and avoidance behavior, and offers approaches to treatment.

Multiple neurotransmitters are potentially involved in the generation of anxiety (Jetty, Charney, \& Goddard, 2001). Glutamate receptors mediate excitatory neurotransmission, and stress activates glutamate transmission in cortical and limbic regions in the CNS. Glutamate receptor antagonists reduce anxiety in animal models. The gamma amino butyric acid (GABA) neurotransmitter is the main inhibitor in the central nervous system and low levels or receptor dysfunction would facilitate arousal. Serotonin has a role in anxiety in animal, but human studies are unclear. Partial agonists such as buspirone and receptor blockers both have clinical benefits in GAD. Corticotrophin releasing factor injection in the CNS in animals causes physiological and behavioral responses similar to stress. Studies with these neurotransmitters and others provide the basis for clinical treatment trials.

Acute flares in COPD frequently cause significant psychological distress secondary to the uncertainty about the course and the severe shortness of breath and could contribute to the development of anxiety through the process referred to as kindling. This kindling model arose from animal studies which demonstrated that progressively smaller amounts of electrical current could induce seizures in mice (Post, Fleming, \& Kapczinsk, 2012; Bender \& Allow, 2011). In other models using stimulants such as cocaine it was noted that animals have a progressive increase in behavioral responses associated with the stimulant drug. Therefore, kindling represents functional and/or structural change in neural activity associated with electrical stimuli (animal models), stress, or drugs and has been used to help understand unipolar depression, bipolar disorder, anxiety, and chronic alcoholism. Patients with COPD with acute exacerbations associated with increased respiratory symptoms, especially dyspnea, often have increased psychological distress (anxiety). If they have repeated exacerbations, they may have a kindling phenomenon in which smaller changes in respiratory distress result in similar or larger changes in anxiety. Dyspnea has both quantitative characteristics (intensity) and behavioral features (unpleasantness) (Parshall et al., 2012). Studies with T-weighted magnetic resonance imaging demonstrate that experimental dyspnea activates the sensorimotor cortex and the supplemental motor area (Von Leupoldt et al., 2008). Dyspnea with unpleasant features activates the right insular cortex and the amygdala. The amygdala has a chemosensor which responds to carbon dioxide and acidosis and initiates fear responses in animals (Ziemann et al., 2009). Activation of these respiratory pathways may also trigger fear and anxiety which in turn amplify the dyspnea. Since these patients typically have a chronic shortness of breath independent of any acute exacerbations, they may eventually have chronic anxiety associated their 
baseline shortness of breath. This is one potential mechanism in which patients with COPD develop a higher prevalence of anxiety. In addition, some medications used in the management of COPD, such as beta-agonists, theophylline, and corticosteroids, cause somatic symptoms secondary to side effects. Finally, patients with COPD often have sleep disturbance which can disrupt circadian rhythms and contribute to development of abnormal psychological responses associated with both internal and external stimuli.

\section{Consequences}

The development of anxiety and GAD can occur in any COPD patient independent of the reduction in pulmonary function and is not preferentially observed in patients with severe disease. Patients with COPD and GAD have more somatic complaints and psychological distress than patients with just COPD. GAD clearly decreases their quality of life and may interfere with chronic medical care. GAD may increase the frequency of acute flares which put patients at immediate risk for mortality and usually cause deterioration in lung function in surviving patients. In addition, there is increased mortality in the year following an acute flare in patients with GAD. Finally, anxiety increases sympathetic activation which can increase cardiovascular morbidity.

\section{Anxiety Treatment}

Patients with GAD frequently have concomitant depression. Therefore, therapeutic decisions must consider the possibility that depression is the more important psychiatric disorder. Treatment approaches to GAD include both medication and psychotherapy (Fricchione, 2004). Tricyclic antidepressants have benefit in patients with GAD but are infrequently used now because of their side effect profile. Selective serotonin reuptake inhibitors such as paroxetine provide significant benefit in patients with GAD. An eight week randomized placebo controlled trial demonstrated that patients on paroxetine had higher response rates $(72 \%)$ than patients on placebo $(56 \%)$. The remission rate increased and the relapse rate $(11 \%$ in those on active therapy vs $41 \%$ on placebo) decreased when the trial was extended 24 weeks in patients who responded to paroxetine (Fricchione, 2004). Serotonin-norepinephrine reuptake inhibitors also provide significant benefit in these patients. The response rates were $58 \%$ at eight weeks and $66 \%$ at six months in patients on active therapy and $36 \%$ and $39 \%$ in those on placebo (Fricchione, 2004). The optimal treatment duration is uncertain but probably should be extended to at least 6 months of treatment. Benzodiazepines are frequently used for acute management, but their long term use should be limited because of side effects and dependence. Patients with GAD also improve with psychotherapy. In particular, cognitive behavioral therapy provides significant benefit within three months, and approximately $40 \%$ of patients improve within six months. In comparison control patients in this study had no benefit at six months. Applied relaxation therapy appears to provide the same benefit as cognitive behavioral therapy.

\section{Anxiety Treatment in COPD}

The management of GAD in patients with COPD is potentially more complicated. These patients are older, often have significant comorbidity, and frequently are on multiple medica- tions. These patients have been treated with selective serotonin reuptake inhibitors with modest benefit. Several studies have suggested that pulmonary rehabilitation improves both anxiety and depression (Coventry, 2009; Cafarella et al., 2012). Coventry recently reviewed this literature and did a meta-analysis on six studies which evaluated responses to rehabilitation (Coventry, 2009). He concluded that rehabilitation significantly improved both anxiety and depression in COPD patients. It is uncertain as to which component of the pulmonary rehabilitation provides this benefit. In addition, it is uncertain as to whether or not patients with severe GAD respond to just pulmonary rehabilitation. Finally, it is unclear as to how long this benefit persists after the completion of pulmonary rehabilitation. However, in general it would seem that the best initial approach to managing GAD in patients with COPD involves comprehensive pulmonary rehabilitation. This should include patient education and, if possible, patient psychotherapy. Patients who stay in maintenance programs should maintain the initial benefits in the reduction of GAD. Patients discharged to home programs can maintain benefit with self-help programs. Patients who have severe GAD who do not respond to pulmonary rehabilitation might benefit from the addition of selective serotonin reuptake inhibitors. This decision may require input from a psychiatrist or psychologist. In addition, physicians need to increase treatment efforts to reduce respiratory symptoms, especially the shortness of breath. Finally, all medications should be reviewed to identify medications causing important side effects, and sleep habits and sleep quality should be reviewed.

\section{Conclusion}

In summary, patients with COPD frequently have GAD. These psychological symptoms decrease the quality of life and potentially contribute to the development of acute flares. There is likely a complex interaction between the symptom of dyspnea and the development of anxiety, and this process involves the limbic system and the amygdala. Medical management should focus on measures which reduce dyspnea and prevent acute flares. Comprehensive pulmonary rehabilitation can reduce anxiety symptoms, but in patients with severe GAD medication may be necessary. This decision probably requires input from a psychologist and/or psychiatrist; the drug of choice is a selective serotonin reuptake inhibitor started at a low dose.

\section{REFERENCES}

American Psychiatric Association (2000). Diagnostic and statistics manual of mental disorders (4th ed.). Arlington, VA: Text Revision (DSM-IV-TR).

Andreas, S., Anker, S. D., Scanlon, P. D., \& Somers, V. K. (2005). Neurohumoral activation as a link to systemic manifestations of chronic lung disease. Chest, 128, 3618-3624. doi: $10.1378 /$ chest. 128.5 .3618

Beck, A. T., \& Steer, R. A. (1996). Beck anxiety inventory: Manual. San Antonio, TX: The Psychological Corporation.

Borak, J., Sliwinski, P., Piasecki, Z., \& Zielinski, J. (1991). Psychological status of COPD patients on long term oxygen therapy. The European Respiratory Journal: Official Journal of the European Society for Clinical Respiratory Physiology, 4, 59-62.

Bender, R. E., \& Allow, L. B. (2011). Life stress and kindling in bipolar disorder: Review of the evidence and integration with emerging biopsychosocial theories. Clinical Psychology Review, 31, 383-398. doi:10.1016/j.cpr.2011.01.004 
Brenes, G. A. (2003). Anxiety and chronic obstructive pulmonary disease: prevalence, impact, and treatment. Psychosomatic Medicine, 65, 963-970. doi:10.1097/01.PSY.0000097339.75789.81

Cafarella, P. A., Effing, T. W., Usmani, Z., \& Frith, P. A. (2012). Treatments for anxiety and depression in patients with chronic obstructive pulmonary disease: A literature review. Respirology, 17, 627-638. doi:10.1111/j.1440-1843.2012.02148.x

Coventry, P. A. (2009). Does pulmonary rehabilitation reduce anxiety and depression in chronic obstructive pulmonary disease? Current Opinion in Pulmonary Medicine, 15, 143-149.

doi:10.1097/MCP.0b013e3283218318

Di Marco, F., Verga, M., Reggente, M., Maria Casanova, F., Santus, P., Blasi F., Allegra, L., \& Centanni, S. (2006). Anxiety and depression in COPD patients: The roles of gender and disease severity. Respiratory Medicine, 100, 1767-1774. doi:10.1016/j.rmed.2006.01.026

Eiser, N., West, C., Evans, S., Jeffers, A., \& Quirk F. (1997). Effects of psychotherapy in moderately severe COPD: A pilot study. The European Respiratory Journal: Official Journal of the European Society for Clinical Respiratory Physiology, 10, 1581-1584. doi:10.1183/09031936.97.10071581

Ferguson, C. J., Stanley, M., Souchek, J., \& Kunik, M. E. (2006). The utility of somatic symptoms as indicators of depression and anxiety in military veterans with chronic obstructive pulmonary disease. Depression and Anxiety, 23, 42-49. doi:10.1002/da.20136

Fricchione, G. (2004). Generalized anxiety disorder. New England Journal of Medicine, 351, 675-682. doi:10.1056/NEJMcp022342

Heindl, S., Lehnert, M., Criee, C.-P., Hasenfuss G., \& Andreas, S. (2001). Marked sympathetic activation in patients with chronic respiratory failure. American Journal of Respiratory and Critical Care Medicine, 164, 597-601.

Hynninen, K. M., Breitve, M. H., Wiborg, A. B., Pallesen, S., \& Nordhus, I. H. (2005). Psychological characteristics of patients with chronic obstructive pulmonary disease: A review. Journal of psychosomatic research, 59, 429-443. doi:10.1016/j.jpsychores.2005.04.007

Hill, K., Geist, R., Goldstein, R. S., \& Lacasse, Y. (2008). Anxiety and depression in end-stage COPD. The European Respiratory Journal: Official Journal of the European Society for Clinical Respiratory Physiology, 31, 667-677. doi:10.1183/09031936.00125707

Jetty, R. V., Charney, D. S., \& Goddard, A. W. (2001). Generalized anxiety disorder. Psychiatric Clinics of North America, 24, 75-97. doi:10.1016/S0193-953X(05)70207-0

Kessler, R. C., Berglund, P., Demler, O., Jin, R., Merikangas, K. R., \& Walters, E. E. (2005). Lifetime prevalence and age-of-onset distributions of DSM-IV disorders in the National Comorbidity Survey Replication. Archives of General Psychiatry, 62, 593-602. doi:10.1001/archpsyc.62.6.593

Kunik, M. E., Roundy, K., Veazey, C., Souchek, J., Richardson, P., Wray, N. P., \& Stanley, M. A. (2005). Surprisingly high prevalence of anxiety and depression in chronic breathing disorders. Chest, 127, 1205-1211. doi:10.1378/chest.127.4.1205

Laurin, C., Moullec, G., Bacon, S. L., \& Lavoie, K. L. (2012). Impact of anxiety and depression on chronic obstructive pulmonary disease exacerbation risk. American Journal of Respiratory and Critical Care Medicine, 185, 918-923. doi:10.1164/rccm.201105-0939PP

Light, R. W., Merrill, E. J., Despars, J. A., Gordon, G. H., \& Mutalipassi, L. R. (1985). Prevalence of depression and anxiety in patients with COPD. Relationship to functional capacity. Chest, 87, 35-38. doi: $10.1378 /$ chest.87.1.35

Parshall, M. B., Schwartzstein, R. M., Adams, L., Banzett, R. B., Manning, H. L., Boubeau, J., Calverley, P. M., Gift, A. G., Harver, A., Lareau, S. C., Mahler, D. A., Meek, P. M., \& O’Donnell, D. E. (2012). An official American Thoracic Society statement: Update on the mechanisms, assessment, and management of dyspnea. American Journal of Respiratory and Critical Care Medicine, 185, 435-452. doi:10.1164/rccm.201111-2042ST

Post, R. M., Fleming, J., \& Kapczinsk, F. (2012). Neurobiological correlates of illness progression in the recurrent affective disorders. Journal of Psychiatric Research, 46, 561-573. doi:10.1016/j.jpsychires.2012.02.004

Salpeter, S. R., Ormiston, T. M., \& Salpeter, E. E. (2004). Cardiovascular effects of $\beta$-agonists in patients with asthma and COPD: A meta-analysis. Chest, 125, 2309-2321. doi:10.1378/chest.125.6.2309

Von Leupoldt, A., Sommer, T., Kegat, S., Baumann,H. J., Klose, H., Dahme, B., \& Buchel, C. (2008). The unpleasantness of perceived dyspnea is processed in the anterior insula and amygdale. American Journal of Respiratory and Critical Care Medicine, 177, 1026-1032. doi:10.1164/rccm.200712-18210C

Wennlund, B. T., \& Carlstrom, K. (2000). Impact of hypoxaemia on neuroendocrine function and catecholamine secretion in chronic obstructive pulmonary disease (COPD). Effects of lung-term oxygen treatment. Respiratory Medicine, 94, 1221-1228.

doi:10.1053/rmed.2000.0953

Xu, W., Collet, J. P., Shapiro, S., Lin, Y., Yang, T., Platt, R. W., Wang, C., \& Bourbeau, J. (2008). Independent effect of depression and anxiety on chronic obstructive pulmonary disease exacerbations and hospitalizations. American Journal of Respiratory and Critical Care Medicine, 178, 913-920. doi:10.1164/rccm.200804-619OC

Yohannes, A. M., Baldwin, R. C., \& Connolly, M. J. (2000). Depression and anxiety in elderly outpatients with chronic obstructive pulmonary disease: Prevalence, and validation of the BASDEC screening questionnaire. International Journal of Geriatric Psychiatry, 15, 1090-1096.

doi:10.1002/1099-1166(200012)15:12<1090::AID-GPS249>3.0.CO; $\underline{2-\mathrm{L}}$

Zandbergen, J., Bright, M., Pols, H., Fernandez, I., de Loof, C., \& Griez, E. J. (1991). Higher lifetime prevalence of respiratory diseases in panic disorder? The American Journal of Psychiatry, 148, 15831585 .

Ziemann, E., Allen, J. E., Dahdaleh, N. S., Drebo, I. I., Coryell, M. W., Wunsch, A. M., Lynch, C. M., Faraci, F. M., Howard, M. A., Welsh, M. J., \& Wemmie, J. A. (2009). The amygdale is a chemosensor that detects carbon dioxide and acidosis to elicit fear behavior. Cell, 139, 1012-1021. doi:10.1016/j.cell.2009.10.029 